\title{
Características sociodemográficas e clínicas de adolescentes candidatos à doação de sangue em um hemocentro
}

Sociodemographic and clinical characteristics of adolescent candidates for blood donation in a hemotherapy service

Características sociodemográficas y clínicas de adolescentes candidatos a donación de sangre en un hemocentro

\section{Fernanda Duarte Siqueira ${ }^{1}$, Nara Marilene Oliveira Girardon-Perlini ${ }^{2}$, Anelise Levay Murari $^{3}$, Carla Tatiana dos Santos Coelho ${ }^{4}$, Lauvir Freitas Carneiro ${ }^{5}$}

\begin{abstract}
Resumo: Objetivo: conhecer as características sociodemográficas e clínicas de adolescentes de 16 e 17 anos candidatos à doação de sangue em um Hemocentro Regional do Estado do Rio Grande do Sul. Método: estudo transversal, descritivo, desenvolvido com base nos registros do banco de dados de um Hemocentro no período de janeiro de 2012 a dezembro de 2016, conforme critério de ampliação de seleção de doadores da Portaria ํㅡ 1.353/ 2011. Resultados: o total de candidatos à doação de sangue entre 16 e 17 anos foi de 473 pessoas, com predomínio de candidatos aptos à doação $(78,2 \%)$, do sexo feminino $(57,5 \%)$, de cor caucasiana $(72,5 \%)$, que doavam pela primeira vez (86\%) de forma voluntária. Conclusão: a possibilidade de doação de sangue, incluindo os adolescentes, necessita ser amplamente divulgada e ações de marketing e de educação em saúde utilizadas como estratégias para sensibilização, captação e fidelização.
\end{abstract}

Descritores: Doadores de sangue; Seleção do doador; Serviço de hemoterapia; Adolescente; Enfermagem

Abstract: Objective: to know the sociodemographic and clinical characteristics of adolescents aged 16 and 17 who are candidates for blood donation in a Regional hemotherapy service in the State of Rio Grande do Sul. Method: cross-sectional, descriptive study, developed based on the records of a hemoterapy service database from January 2012 to December 2016, according to the criteria for expanding the selection of donors in Ordinance $\mathrm{N}^{\circ}$.

\footnotetext{
${ }^{1}$ Enfermeira. Mestra em Enfermagem. Doutoranda no Programa de Pós-Graduação em Enfermagem da Universidade Federal de Santa Maria (UFSM). Santa Maria, RS, Brasil. E-mail: fesiqueiraenf@gmail.com ORCID: https://orcid.org/0000-0002-1157-1779

2 Enfermeira. Pós-Doutora em Enfermagem. Professora do Departamento de Enfermagem. UfSM. Santa Maria, RS, Brasil. E-mail: nara.girardon@gmail.com ORCID: https://orcid.org/0000-0002-3604-2507

3 Farmacêutica. Doutora em Farmacologia. Professora do Departamento de Morfologia. UFSM. Santa Maria, RS, Brasil. E-mail: aneliselm@gmail.com ORCID: https://orcid.org/0000-0001-7075-173X

4 Bióloga. Diretora do Hemocentro Regional de Santa Maria. Santa Maria, RS, Brasil. E-mail: carlahemo@hotmail.com ORCID: https://orcid.org/0000-0001-8606-5056

${ }^{5}$ Estudante do Ensino Médio Educação de Jovens e Adultos. Instituto Estadual de Educação Olavo Bilac. Bolsista de Iniciação Científica Ensino Médio (2016-2017) da UFSM. E-mail: Freitas.lauvir6@gmail.com ORCID: https://orcid.org/0000-0001-7894-0949
} 
1.353 / 2011. Results: the total number of blood donation candidates between 16 and 17 years old was 473 people, with a predominance of candidates suitable for donation (78.2\%), female (57.5\%), Caucasian (72.5\%), who donated for the first time $(86 \%)$ voluntarily. Conclusion: the possibility of blood donation, including adolescents, needs to be widely publicized and marketing and health education actions used as strategies for raising awareness, attracting and retaining customers.

Descriptors: Blood donors, Donor selection, Hemotherapy service, Adolescent, Nursing

Resumen: Objetivo: Conocer las características sociodemográficas y clínicas de los adolescentes de 16 y 17 años candidatos para la donación de sangre en un servicio de hemoterapia en el Estado de Rio Grande do Sul. Método: Estudio descriptivo transversal, desarrollado en base a los registros de una base de datos de servicio de hemoterapia desde enero de 2012 hasta diciembre de 2016, de acuerdo con los criterios para ampliar la selección de donantes en la Ordenanza $N^{\circ}$ 1.353/2011. Resultados: El número total de candidatos para donación de sangre entre 16 y 17 años fue 473 personas, con predominio de candidatos aptos para la donación (78,2\%), mujeres (57,5\%), caucásicas $(72,5 \%)$, que donó por primera vez (86\%) voluntaria. Conclusión: la posibilidad de donación de sangre, incluidos los adolescentes, debe ser ampliamente publicitada y las acciones de marketing y educación sanitaria deben usarse como estrategias para crear conciencia, atraer y retener clientes.

Descriptores: Donantes de sangre, Selección de donantes, Servicio de hemoterapia, Adolescente, Enfermería.

\section{Introdução}

A falta de hemocomponentes constitui-se em preocupação de caráter mundial por não haver elementos que possam substituí-los. De acordo com o Ministério da Saúde estima-se que no Brasil sejam realizados cerca de três milhões e seiscentos mil transfusões de sangue todos os anos. Apenas $1,9 \%$ da população doa sangue com regularidade. Além disso, acredita-se que Estados com maior desenvolvimento tecnológico, como Rio de Janeiro, São Paulo e Rio Grande do Sul apresentem maior demanda por sangue. ${ }^{1}$ Neste sentido, para manter os estoques é necessário que $3 \%$ a $5 \%$ da população seja doadora fidelizada..$^{2-3}$

No intuito de motivar à consciência coletiva de responsabilidade, compromisso e solidariedade o Ministério da Saúde em 2011, por meio da Agência Nacional de Vigilância Sanitária (Anvisa), emitiu a Portaria nº 1.353, de 13 de junho, que amplia os critérios de seleção de doadores permitindo a inclusão de adolescentes de 16 e 17 anos, desde que com o consentimento formal dos pais ou responsáveis legais. Esse propósito visa ao aumento do contingente de doadores de sangue e hemoderivados. ${ }^{4}$ 
Dentre os tipos de doação estão à voluntária ou espontânea, a de reposição, a autóloga e a aférese. A voluntária ou espontânea caracteriza-se como doações de uma unidade de sangue ou de um de seus componentes motivadas por um ato altruísta para manter os estoques dos serviços de hemoterapia sem a identificação do nome do possível receptor. A reposição tem o objetivo de repor o estoque do banco de sangue, sendo feita por indivíduos que doam por motivos pessoais, a partir da necessidade apresentada por algum membro da família ou conhecido. Autóloga é realizada pelo doador para si mesmo e a aférese consiste na retirada de apenas um componente do sangue total. ${ }^{2-3}$

Antes de proceder à coleta de sangue os candidatos à doação passam por uma triagem clínica que consiste na avaliação da história clínica e epidemiológica do estado atual de saúde, de hábitos e comportamentos e doenças que os inabilitem como doadores. Esta estratégia contribui para determinar se o candidato está em condições de doar sangue sem que haja prejuízo à saúde do doador e do receptor. Para aqueles considerados aptos, após a coleta, é realizada, ainda, a análise laboratorial do sangue, antes da liberação dos hemocomponentes para transfusão. Essas medidas visam a proteger e garantir a segurança do binômio doador-receptor e a consequente melhora na qualidade do sangue a ser transfundido, diminuindo, assim, os riscos inerentes à terapêutica transfusional e à transmissão de doenças infecciosas. ${ }^{2-3}$

As competências e atribuições da equipe de enfermagem nos serviços hemoterápicos têm o respaldo do Conselho Federal de Enfermagem, por meio da Resolução N.306/2006 (CONFEN, 2006). ${ }^{5}$ A enfermagem tem contato direto com o candidato à doação, orientando sobre todas as etapas da doação de sangue e as possíveis reações adversas durante e após a coleta de sangue. Cabe ao enfermeiro, especificamente, realizar a triagem clínica, por meio de entrevista com o provável doador para avaliar os antecedentes e o estado atual em ambiente que garanta a privacidade e o sigilo das informações prestadas. Para mais, o enfermeiro, desempenha uma função importante na conscientização da equipe de enfermagem para um cuidado humanizado e 
seguro, que garanta qualidade e confiança ao doador no processo de captação, estimulando a fidelização de doadores. ${ }^{6}$

Portanto, considerando que a possibilidade de adolescentes de 16 e 17 anos doarem sangue, é pouco divulgada e que pouco se conhece acerca de como estes indivíduos têm se comportado após sua inclusão na regulamentação ministerial, destaca-se a relevância de estudos que explorem a participação deste grupo populacional no contexto da doação de sangue. Pesquisas dessa natureza se justificam pela atribuição que compete ao enfermeiro de atuar no planejamento, execução, coordenação, supervisão e avaliação de procedimentos que envolvem a hemoterapia. ${ }^{5}$ Nesse sentido, destaca-se sua importância no processo que visa à conscientização e sensibilização para a captação de doadores, propondo, inclusive, estratégias educativas que possam incentivar e melhor informar o público-alvo, favorecendo esse exercício de solidariedade e cidadania. ${ }^{7}$

A doação de sangue por adolescentes de 16 e 17 anos ainda é uma temática incipiente no Brasil. Em busca realizada em base de dados online, via portal regional da Biblioteca Virtual em Saúde (BVS), utilizando-se os descritores “adolescentes and doadores de sangue” localizou-se uma produção no contexto nacional, evidenciando uma lacuna no conhecimento, uma vez que o estudo buscou avaliar o impacto da ampliação da faixa etária elegível para doação no perfil dos doadores e no número de doações. Os resultados obtidos concluíram que tal medida não aumentou a proporção de doadores na população. ${ }^{8}$ Contudo, as características sociodemográficas e clínicas dos doadores não foram consideradas na investigação.

Sendo assim, a presente investigação pretende responder a seguinte questão: quais as características sociodemográficas e clínicas de adolescentes de 16 e 17 anos candidatos à doação de sangue em um Hemocentro Regional? 
5 | Siqueira FD, Girardon-Perlini NMO, Murari AL, Coelho CTS, Carneiro LF

Assim, tem-se como objetivo: conhecer as características sociodemográficas e clínicas de adolescentes de 16 e 17 anos candidatos à doação de sangue em um Hemocentro Regional do Estado do Rio Grande do Sul.

\section{Método}

Trata-se de um estudo transversal e descritivo, desenvolvido com base nos registros disponíveis no banco de dados eletrônico do Hemocentro Regional de Santa Maria (HEMOVIDA), de candidatos à doação de sangue e hemocomponentes entre o período $1^{\circ}$ de janeiro de 2012 a 31 de dezembro de 2016.

As informações para realização desta investigação foram extraídas do banco de dados do HEMOVIDA, contemplando as seguintes variáveis disponíveis: ano, sexo, idade, cor da pele autorrelatada, candidatos aptos e inaptos, tipo de doador (primeira vez ou de repetição) tipo de doação (voluntária ou espontânea e reposição) e motivos de inaptidão como anemia, desistência, hipotensão arterial, acesso venoso inadequado e outras causas não especificadas nos registros disponíveis. A coleta dessas informações ocorreu no mês de maio de 2017. Quanto aos critérios de seleção foram incluídos os registros de adolescentes de 16 e 17 anos de idade e excluídos os registros incompletos.

Cabe destacar que esses dados são autorreferidos pelos candidatos, no momento em que se apresentam para fazer doação de sangue, e informados no Sistema pelos profissionais do Hemocentro. Trata-se de um formulário padrão implantado em 2010 e utilizado por todos os Hemocentros do Rio Grande do Sul, sendo que os dados disponíveis no HEMOVIDA referem-se ao Estado. Contudo, para o presente estudo foram disponibilizadas somente as informações referentes ao Hemocentro Regional de Santa Maria.

Assim, a partir das informações de interesse extraídas, os dados foram digitados e organizados em uma planilha eletrônica, no programa Microsoft Office Excel ${ }^{\circledR} 2010$. A análise 
das variáveis categóricas e quantitativas foi realizada por meio da estatística descritiva (números absolutos e percentuais).

A referida pesquisa integra uma investigação maior, intitulada "Captação de doadores voluntários de sangue e hemocomponentes: contribuições de uma equipe multiprofissional”, que foi aprovada pelo Comitê de Ética em Pesquisa sob Parecer número 1.189.482. O referido estudo foi realizado junto ao Hemocentro Regional de Santa Maria e buscou avaliar o resultado de estratégias implementadas por uma equipe multiprofissional para ampliar a captação de doadores de sangue e hemocomponentes em geral. No presente estudo é apresentado um recorte descritivo dos dados referentes aos doadores adolescentes de 16 e 17 anos, especificamente.

Ressalta-se que foram observados todos os preceitos éticos que regem pesquisas envolvendo seres humanos, em conformidade com a Resolução 466/12 e que o estudo contou com o apoio e aprovação da gerência administrativa do Hemocentro Regional para realização do mesmo.

\section{Resultados}

Dos registros encontrados, no período de 2012 a 2016, o número de candidatos à doação de sangue total, entre 16 a 69 anos de idade, foi de 55.346 indivíduos. Destes 47.401 (85,6\%) foram considerados aptos para doação e 7.945 (14,4\%) inaptos. Diante disso, integraram a presente investigação 473 registros daqueles candidatos à doação com idade entre 16 e 17 anos. Estes representaram $0,85 \%$ do total de candidatos à doação.

Sequencialmente, na Tabela 1, é explicitada a distribuição de candidatos com idade entre 16 e 17 anos à doação de sangue considerados aptos e inaptos. Nesta, constata-se que o maior percentual, no geral, encontra-se entre os candidatos aptos a doação de sangue com $78,2 \%$ $(n=370)$. 
7 | Siqueira FD, Girardon-Perlini NMO, Murari AL, Coelho CTS, Carneiro LF

Tabela 1 - Candidatos considerados aptos e inaptos para doação de sangue, no período de 2012 a 2016. Santa Maria, RS, 2017.

\section{Candidatos à doação}

(16 e 17 anos)

\begin{tabular}{ccccccc}
\hline \multirow{2}{*}{ Ano } & \multicolumn{2}{c}{ Aptos } & \multicolumn{2}{c}{ Inaptos } & \multicolumn{2}{c}{ Total } \\
& $\mathbf{n}$ & $(\mathbf{\%})$ & $\mathbf{n}$ & $(\mathbf{\%})$ & $\mathbf{n}$ & $(\mathbf{\%})$ \\
\hline 2012 & 49 & $(75,4)$ & 16 & $(24,6)$ & 65 & $(100)$ \\
2013 & 67 & $(84,8)$ & 12 & $(15,2)$ & 79 & $(100)$ \\
2014 & 90 & $(79,7)$ & 23 & $(20,3)$ & 113 & $(100)$ \\
2015 & 91 & $(77,8)$ & 26 & $(22,2)$ & 117 & $(100)$ \\
2016 & 73 & $(73,7)$ & 26 & $(26,3)$ & 99 & $(100)$ \\
\hline Total & $\mathbf{3 7 0}$ & $(\mathbf{7 8 , 2}$ & $\mathbf{1 0 3}$ & $(\mathbf{2 1 , 8})$ & $\mathbf{4 7 3}$ & $(\mathbf{1 0 0})$ \\
\hline
\end{tabular}

Fonte: Banco de dados HEMOVIDA do Hemocentro Regional de Santa Maria/RS

A Tabela 2 apresenta a distribuição dos candidatos à doação segundo o sexo e o período de comparecimento dos adolescentes. Desse modo, verifica-se que os candidatos que mais doaram foram do sexo feminino com $57,51 \%(n=272)$.

Tabela 2 - Distribuição dos candidatos de acordo como o sexo e o ano de doação (2012 a 2016). Santa Maria, RS, 2017.

\begin{tabular}{|c|c|c|c|c|c|c|}
\hline \multirow{3}{*}{ Ano } & \multicolumn{4}{|c|}{ Sexo } & \multirow{2}{*}{\multicolumn{2}{|c|}{ Total }} \\
\hline & \multicolumn{2}{|c|}{ Feminino } & \multicolumn{2}{|c|}{ Masculino } & & \\
\hline & $\mathbf{N}$ & $(\%)$ & $\mathbf{N}$ & $(\%)$ & $\mathbf{n}$ & $(\%)$ \\
\hline 2012 & 39 & $(8,25)$ & 26 & $(5,50)$ & 65 & $(13,74)$ \\
\hline 2013 & 44 & $(9,30)$ & 35 & $(7,40)$ & 79 & $(16,70)$ \\
\hline 2014 & 58 & $(12,26)$ & 55 & $(11,63)$ & 113 & $(23,89)$ \\
\hline 2015 & 68 & $(14,38)$ & 49 & $(10,36)$ & 117 & $(24,74)$ \\
\hline 2016 & 63 & $(13,32)$ & 36 & $(7,61)$ & 99 & $(20,93)$ \\
\hline Total & 272 & $(57,51)$ & 201 & $(42,49)$ & 473 & $(\mathbf{1 0 0})$ \\
\hline
\end{tabular}

Fonte: Banco de dados HEMOVIDA do Hemocentro Regional de Santa Maria/RS.

$\mathrm{Na}$ Tabela 3, é evidenciada a distribuição dos candidatos em relação ao tipo de doador e doação no período de 2012 a 2016. Ao analisar os candidatos quanto ao tipo de doador, verificase em predominância os indivíduos que doaram de primeira vez. No que tange ao tipo de doação destaca-se os candidatos que doaram voluntária ou espontaneamente. 
Tabela 3 - Distribuição dos candidatos à doação conforme o tipo de doador e doação com relação à periodicidade de comparecimento do doador. Santa Maria, RS, 2017

\begin{tabular}{|c|c|c|c|c|c|c|c|}
\hline \multirow{3}{*}{ Ano } & \multirow{3}{*}{ Tipo de doador } & \multicolumn{4}{|c|}{ Tipo de doação } & & \multirow[b]{3}{*}{$(\%)$} \\
\hline & & \multicolumn{2}{|c|}{$\begin{array}{c}\text { Voluntária } \\
\text { (Espontânea) }\end{array}$} & \multicolumn{2}{|c|}{ Reposição } & & \\
\hline & & $\mathbf{n}$ & $(\%)$ & $\mathbf{n}$ & $(\%)$ & $\mathbf{n}$ & \\
\hline \multirow{3}{*}{2012} & $1^{\mathrm{a}}$ Vez & 50 & $(10,57)$ & 9 & $(1,90)$ & 59 & $(12,47)$ \\
\hline & Repetição & 5 & $(1,06)$ & 1 & $(0,21)$ & 6 & $(1,27)$ \\
\hline & Total & 55 & $(11,63)$ & 10 & $(2,11)$ & 65 & $(13,74)$ \\
\hline \multirow{3}{*}{2013} & $1^{\mathrm{a}} \mathrm{Vez}$ & 59 & $(12,47)$ & 16 & $(3,38)$ & 75 & $(15,86)$ \\
\hline & Repetição & 4 & $(0,85)$ & - & - & 4 & $(0,85)$ \\
\hline & Total & 63 & $(13,32)$ & 16 & $(3,38)$ & 79 & $(16,70)$ \\
\hline \multirow{3}{*}{2014} & $1^{\mathbf{a}} \mathrm{Vez}$ & 72 & $(15,22)$ & 20 & $(4,23)$ & 92 & $(19,45)$ \\
\hline & Repetição & 16 & $(3,38)$ & 5 & $(1,06)$ & 21 & $(4,44)$ \\
\hline & Total & 88 & $(18,60)$ & 25 & $(5,29)$ & 113 & $(23,89)$ \\
\hline \multirow{3}{*}{2015} & $1^{\mathbf{a}} \mathrm{Vez}$ & 75 & $(15,86)$ & 26 & $(5,50)$ & 101 & $(21,35)$ \\
\hline & Repetição & 14 & $(2,96)$ & 2 & $(0,42)$ & 16 & $(3,38)$ \\
\hline & Total & 89 & $(18,82)$ & 28 & $(5,92)$ & 117 & $(24,74)$ \\
\hline \multirow{4}{*}{2016} & $1^{\mathrm{a}} \mathrm{Vez}$ & 59 & $(12,47)$ & 23 & $(4,86)$ & 82 & $(17,34)$ \\
\hline & Repetição & 14 & $(2,96)$ & 3 & $(0,63)$ & 17 & $(3,59)$ \\
\hline & Total & 73 & $(15,43)$ & 26 & $(5,50)$ & 99 & $(20,93)$ \\
\hline & Total Geral & 368 & $(77,80)$ & 105 & $(22,20)$ & 473 & $(100)$ \\
\hline
\end{tabular}

Fonte: Banco de dados HEMOVIDA do Hemocentro Regional de Santa Maria/RS. $\mathrm{P}<0,05$ significativo.

Finalizando a apresentação dos dados, na Tabela 4 está explicitada a distribuição dos candidatos à doação de sangue em relação à cor da pele. Nesta, identifica-se que a maioria dos adolescentes eram da cor caucasiana $72,5 \%(n=341)$.

Tabela 4 - Distribuição dos candidatos à doação quanto à cor da pele. Santa Maria, RS, 2017.

\begin{tabular}{|c|c|c|c|c|c|c|}
\hline \multirow[b]{2}{*}{ Ano } & \multicolumn{4}{|c|}{ Cor da Pele } & \multirow[b]{2}{*}{ Mestiça } & \multirow[b]{2}{*}{ Total } \\
\hline & Caucasiana & Caucasiana B. ${ }^{*}$ & Negra & Indígena & & \\
\hline 2012 & $56(86,1)$ & $4(6,2)$ & - & $1(1,5)$ & $4(6,2)$ & 65 \\
\hline 2013 & $51(64,5)$ & $21(26,6)$ & $3(3,8)$ & - & $4(5,1)$ & 79 \\
\hline 2014 & $76(67,8)$ & $24(21,4)$ & $1(1)$ & - & $11(9,8)$ & 112 \\
\hline 2015 & $75(64)$ & $25(21,4)$ & $8(6,9)$ & - & $9(7,7)$ & 117 \\
\hline 2016 & $83(85,6)$ & $3(3,1)$ & $3(3,1)$ & - & $8(8,2)$ & 97 \\
\hline Total & $341(72,5)$ & $77(16,4)$ & $15(3,2)$ & $1(0,2)$ & $36(7,7)$ & 470 \\
\hline
\end{tabular}

* Caucasiana brasileira

Fonte: Banco de dados HEMOVIDA do Hemocentro Regional de Santa Maria/RS. 


\section{Discussão}

As informações coletadas do banco de dados HEMOVIDA representam os resultados de um centro regional de doação de sangue que no período investigado apresentou uma tendência de discreto aumento de doações entre os adolescentes de 16 e 17 anos no sexo feminino, cor caucasiana, aptos, que doaram pela primeira voluntária ou espontaneamente. Estudo realizado na cidade de Tubarão, no Estado de Santa Catarina, Brasil, identificou que do total de doações, $0,29 \%$ em 2011 e $0,49 \%$ em 2013 corresponderam a adolescente de 16 a 18 anos. $^{8}$

Quanto ao consentimento dos pais ou responsáveis legais, salienta-se que a autorização deve cumprir todas as exigências e responsabilidades prenunciadas aos demais doadores, ou seja, submeter-se a triagem clínica, realizar e receber os resultados da análise laboratorial. No entanto, os resultados dos testes de triagem laboratorial somente poderão ser cedidos ao próprio doador adolescente. ${ }^{9}$ A necessidade de consentimento formal de pais ou responsável para a doação, pode constituir-se em um fator que restrinja o número de candidatos adolescente à doação.

A maioria dos adolescentes, cujos dados compuseram este estudo, foram considerados aptos à doação de sangue. Cabe destacar que a inaptidão pode ser definida como temporária, quando o candidato se encontra impedido de doar sangue por determinado período, ou definitiva, para aqueles que nunca poderão doar sangue. ${ }^{2}$ Quanto aos fatores que levaram à inaptidão, $77,7 \%(\mathrm{n}=80)$ das causas não estavam descritas no sistema, sendo classificadas como “outras causas”, 14,6\% $(\mathrm{n}=15)$ foi por anemia, 3,9\% $(\mathrm{n}=4)$ desistências, $2,9 \%(\mathrm{n}=3)$ por hipotensão arterial e 0,9\% (n=1) devido a acesso venoso inadequado. Informações detalhadas, que discriminem os motivos de inaptidão presente dentre as "outras causas" pode sinalizar para a necessidade de aprimorar, no sistema, o processo de registro dos dados, representando a 
realidade do serviço para que se possa investir na redução do número de adolescentes inaptos. ${ }^{10-}$ 11

O fato dos adolescentes terem a iniciativa de doar sangue, e serem considerados aptos, pode motivá-los a continuarem com este ato, independente se o fizerem pelo espírito altruísta ou social, de fazer o bem a alguém próximo ou por ajudar o outro. Além disso, pode favorecer a captação de outros adolescentes e, consequentemente, aumentar o quantitativo de sangue e hemoderivados nos centros de doação. ${ }^{10}$ Identificar algum impedimento, no processo de triagem, que impossibilite tornar-se doador tem sido apontado como um fator que favorece o comportamento negativo para a não doação, desestimula a atitude e faz com que a pessoa multiplique essa opinião em seu grupo de convivência. ${ }^{12}$

Por outro lado, dentre os motivos de inaptidão podem ser considerados, além de doenças que impeçam a doação, os resultado do estilo de vida e dos hábitos das pessoas que, no caso dos adolescentes, podem estar relacionados a fatores como a vulnerabilidade ante comportamentos sexuais de riscos, ingestão de bebidas alcoólicas, padrão alimentar inadequado que pode levar à anemia, gravidez, uso de drogas e falta de informação a respeito do processo de doação de sangue. ${ }^{11-13}$ Neste contexto, realizar atividades educativas, sobretudo em escolas, com o intuito de instigar os adolescentes, por meio da oferta de informações efetivas, sobre hábitos saudáveis e o processo de doação de sangue, pode contribuir para desenvolver a conscientização em relação a esses aspectos, favorecendo ao aumento de candidatos à doação e, consequentemente, à diminuição de inaptidão na triagem clínica. Iniciativas dessa natureza sensibilizam para tornar a doação um comportamento habitual entre os adolescentes, consciente e responsável que, além de ser um dos objetivos das políticas de saúde, é um compromisso social. ${ }^{11}$

Os adolescentes do sexo feminino representaram a maioria dos candidatos à doação de sangue. Esse resultado vai ao encontro de investigação conduzida em um Hemocentro público no município de Fortaleza, no Estado do Ceará, com candidatos de 16 e 17 anos, no ano de 2011, 
11 | Siqueira FD, Girardon-Perlini NMO, Murari AL, Coelho CTS, Carneiro LF

o qual identificou que $63,4 \%$ dos doadores eram do sexo feminino. ${ }^{13}$ Constatação semelhante também é apontada em estudo realizado em Estocolmo/Suécia, no qual 60\% eram candidatos do sexo feminino. ${ }^{14} \mathrm{~A}$ maior frequência de doadores femininos pode estar relacionada ao fato de as mulheres serem mais sensíveis à questão humanitária da doação de sangue e ter mais propensão a procurar o serviço após alguma propaganda ou palestra. ${ }^{15} \mathrm{Em}$ comparação com os resultados da distribuição nacional de doadores de sangue, no período de 2012 a 2015, os candidatos do sexo masculino apresentaram maiores percentuais, porém, ressalta-se que os adolescentes entre 16 e 17 anos não foram considerados nessa investigação. ${ }^{2-3}$

No que se refere ao tipo de doador este estudo aponta como predominante os doadores de primeira vez, o que mostra o interesse dos adolescentes em buscarem os serviços de hemoterapia para doação. Embora a literatura não explore suficientemente os motivos pelos quais os adolescentes decidem procurar os serviços de coleta de sangue pela primeira vez, considera-se que estes não diferem do elencado pela população adulta e que referem a necessidades de sangue por parte de familiares hospitalizados, a influencia de amigos doadores e o marketing das campanhas em prol da doação. ${ }^{12,15}$

Como no tipo de doação predominaram os candidatos que doaram voluntariamente, pode-se inferir que alguns destes podem ter sido acessados em atividades de sensibilização para captação de doadores de sangue. Dentre essas atividades, destacam-se as campanhas, realizadas pelas instituições de saúde e pelo poder público, veiculadas principalmente na televisão, para estimular a população a doar sangue..$^{16-17}$

As estratégias de marketing desenvolvidos em campanhas e em ações educativas visam informar, sensibilizar e estimular a população e os adolescentes à doação de sangue de primeira vez, bem como a se tornarem doadores fidelizados/regulares. Em relação a isso a Organização Mundial da Saúde ressalta a importância dos doadores de repetição, em que a coleta de sangue apresenta baixo índice de rejeição por sorologia positiva e comportamento de risco, sendo, por 
isso, a mais necessária e recomendada, considerando-se o abastecimento de sangue mais seguro e sustentável nos serviços de hemoterapia. ${ }^{15}$

De forma positiva as campanhas são vistas favoravelmente por tentar atrair a população, conseguindo dar visibilidade ao problema e disseminar informações pela mídia. ${ }^{19}$ Entretanto, as campanhas, por si, apresentam limitações quanto à sua eficácia decorrentes da fragilidade em esclarecer adequadamente os requisitos para ser um doador e em tratar os medos relacionados à doação de sangue. Sendo assim, é necessário buscar novas formas de abordar a população, promovendo uma cultura propícia à doação. Para isso é importante incluir discussões relacionadas a esse tema na educação das crianças nas escolas, uma vez que este ambiente exerce influência na aquisição de valores e estimula o exercício da cidadania, transformando os indivíduos não somente em doadores, mas também em multiplicadores dessa perspectiva. ${ }^{17}$

Em relação à cor da pele autorreferida pelos adolescentes candidatos à doação prevaleceu os indivíduos de cor caucasiana e caucasiana brasileira. Neste sentido, a cor caucasiana brasileira incluem os pardos e indivíduos de origem miscigenada. O Brasil é um país abundantemente miscigenado, o que explica a presença e prevalência de hemoglobinas variantes, que pode estar relacionada com a cor da pele dos doadores de sangue. ${ }^{18-20}$ Dentre as variantes, a hemoglobina $S(\mathrm{HbS})$ é uma das alterações hematológicas hereditárias de maior frequência e está intimamente relacionada a imigração africana, sendo a sua prevalência maior nos seus descendentes. A homozigose para a $\mathrm{HbS}$ (hb SS) resulta na anemia falciforme que impede a doação de sangue. Já a heterozigose (hb AS) é caracterizada pelo traço falciforme que no Brasil varia de 2 a $6 \%$ e seus portadores não apresentam sintomas pela obstrução de pequenos vasos sanguíneos sob condições fisiológicas. ${ }^{21}$ A pesquisa de hemoglobulina S é obrigatória na triagem de doadores de sangue e o concentrado de hemácias provenientes de doador com o traço falciforme não pode ser transfundido em pacientes com hemoglobinopatias, acidose grave, 
hipotermia, recém-nascidos e também em transfusões intrauterinas e procedimentos cirúrgicos com circulação extracorpórea. ${ }^{20-21}$

Considerando-se a distribuição do total de adolescentes que buscaram o Hemocentro para doar sangue no período analisado, nota-se que houve um discreto aumento no número de candidatos á doação a cada ano, tendo retrocedido, porém, no último ano, o que pode estar relacionado ao conteúdo das campanhas realizadas, enfatizando a possibilidade de doação por indivíduos nessa faixa etária. Contudo, evidencia-se que ações voltadas à captação de doadores de sangue precisam ser realizadas constantemente. ${ }^{22}$

\section{Conclusão}

Os resultados identificaram as principais características sociodemográficas e clínicas de adolescentes de 16 e 17 anos candidatos à doação de sangue, entre 2012 a 2016, disponíveis no banco de dados de um Hemocentro Regional do Rio Grande do Sul. Constatou-se que estes indivíduos representaram $0,85 \%$ do total de candidatos. Dentre os adolescentes que buscaram o Serviço, predominaram os do sexo feminino, com cor da pele caucasiana, considerados aptos à doação e que faziam a doação pela primeira vez, de forma voluntária ou espontaneamente.

Ressalta-se que a possibilidade de doação de sangue por adolescentes de 16 e 17 anos necessita ser ampla e reiteradamente divulgada, pautando-se em ações que, não apenas alertem para a importância de doar sangue, mas que sejam educativas em relação aos aspectos que envolvem a doação, os possíveis motivos para a inaptidão e esclareçam dúvidas específicas dessa população.

Para que a doação se torne um comportamento frequente entre os adolescentes, faz-se necessário sensibilizá-los para a doação e a fidelização. Nesse sentido, campanhas em parceria com escolas para divulgação entre os estudantes e os pais - já que estes precisam consentir com a doação e podem ser potenciais candidatos, com apoio da rede de atenção primária a saúde e os 
hemocentros podem colaborar para aumentar o número de doadores, contribuindo para a manutenção dos estoques de hemocomponentes. Outra possibilidade para prover uma cultura de doação de sangue, como um ato de cidadania e solidariedade, refere-se a incluir este tema no contexto curricular da educação básica, podendo ser abordado no estudo do sistema circulatório.

Os resultados deste estudo acrescem ao conhecimento de enfermagem por explorar uma temática ainda incipiente. Também podem contribuir para a prática na área da enfermagem hemoterápica, tendo em vista que o enfermeiro desempenha um papel importante tanto no desenvolvimento de ações para divulgar, fortalecer e incentivar a doação de sangue, como na fidelização de doadores. Nesse sentido, conhecer as características dos adolescentes doadores pode subsidiar a elaboração de protocolos de atendimento, bem como a seleção de estratégias educativas e de marketing direcionados a essa população específica.

Como limitações desta investigação destacam-se o contexto da investigação, que se refere aos dados disponibilizados por um serviço público regional de processamento de sangue e o delineamento adotado no estudo. Sugere-se que novas investigações, especialmente de base populacional e com ampliação das variáveis de interesse, sejam realizadas com o intuito de ampliar o conhecimento da realidade em outros hemocentros e em âmbito nacional quanto à doação de sangue por adolescentes. Por fim, estudos que busquem conhecer acerca das motivações que levam os adolescentes a se candidatarem como doadores e a permanecerem (ou não) são necessários para que possamos entender como interagir com esse grupo e assim desenvolver adequadas estratégias de marketing e campanhas. 


\section{Referências}

1. Ministério da Saúde (BR), Agência Nacional de Vigilância Sanitária (ANVISA). Marco conceitual e operacional de hemovigilância: guia para hemovigilância no Brasil. [Internet] 2015 [acesso em $2019 \mathrm{dez}$ 10]. Disponível em: http://portal.anvisa.gov.br/documents/33868/404938/Marco+Conceitual+e+Operacional+de+Hemovigilânci a+-+Guia+para+a+Hemovigilância+no+Brasil/495fd617-5156-447d-ad22-7211cdbab8a7

2. Ministério da Saúde (BR). Portaria ${ }^{\circ} 158$, de 04 de fevereiro de 2016. Redefine o regulamento técnico de procedimentos hemoterápicos [Internet]. Brasília (DF): Ministério da saúde; 2016 [acesso em 2019 dez 19]. Disponível em: https://cevs.rs.gov.br/upload/arquivos/201612/16112320-portaria-158-de-04-02-2016.pdf

3. Ministério da Saúde (BR), Agência Nacional de Vigilância Sanitária (ANVISA). Relatório dos dados de produção hemoterápica brasileira [Internet]. Brasília (DF): Ministério da Saúde; 2015 [acesso 2019 mar 15]. Disponível em: http://portal.anvisa.gov.br/documents/4048533/4993603/6\%C2\%B0+Boletim+de+Produ\%C3\%A7\%C3\%A3o+ Hemoter\%C3\%A1pica+-+Hemoprod+2017/15545fd5-ad1f-4b00-9340-a811aa910bbc

4. Ministério da Saúde (BR). Portaria nº1.353, de 13 de junho de 2011, aprova o regulamento técnico de procedimentos hemoterápicos [Internet]. Brasília (DF): Ministério da Saúde; 2011 [acesso em 2019 nov 26]. Disponível em: http://www.saude.mg.gov.br/images/documentos/PORTARIA\%20No\%201353\%20de\%2013\%20de\%20junh o\%20de\%202011.pdf

5. Conselho Federal de Enfermagem (COFEN). Resolução $n^{\circ} 511 / 2016$. Aprova a norma técnica que dispõe sobre a atuação de enfermeiros de técnicos de enfermagem em hemoterapia [Internet]. Brasília (DF): COFEN; 2016 [acesso em 2019 dez 13]. Disponível em: http://www.cofen.gov.br/resolucao-cofen-no05112016_39095.html

6. Locks MOH, Salum NC, Barros BS, Matos E, Anders JC, Schneider DG. Perfil dos doadores de sangue que apresentam reações adversas à doação. Rev Bras Enferm [Internet]. 2019 [acesso em 2019 nov 20];72(1):87-94. Disponível em: http://www.scielo.br/scielo.php?script=sci_arttext\&pid=S003471672019000100081\&lng=pt\&nrm=iso\&tlng=pt doi: 10.1590/0034-7167-2018-0305

7. Carneiro LF, Towsed CR, Murari AL. Jovens entre 16 e 17 anos podem doar sangue: um exercício de solidariedade e cidadania. In: 31 ${ }^{\text {a }}$ Jornada Acadêmica Integrada (JAI); 2016 out 17-21; Santa Maria (RS): Universidade Federal de Santa Maria; 2016.

8. Medeiros AN, Mapelli LP, Iser BPM, Goelzer B. Novos critérios para doações de sangue no Brasil: impacto no perfil dos doadores e na quantidade de doações realizadas em uma unidade de coleta de Santa Catarina. Rev AMRIGS [Internet]. 2016 [acesso em 2019 dez 24];60(3):173-7. Disponível em: https://pesquisa.bvsalud.org/portal/resource/pt/biblio-832113 
9. Nascimento AA, Ilha S, Marzari CK, Diefenbach GD, Backes DS. Cuidado de enfermagem no processo de doação de sangue: percepção dos profissionais e dos doadores. Rev Enferm Cent-Oeste Min [Internet]. 2015 [acesso em 2019 nov 23];5(1):1497-504. Disponível em: http://www.seer.ufsj.edu.br/index.php/recom/article/view/555/839

10. Ministério da Saúde (BR), Agência Nacional de Vigilância Sanitária (ANVISA). 4º Boletim de Produção Hemoterápica. Brasília (DF): Ministério da Saúde; 2017.

11. Vieira GNT, Sousa FES, Barbosa DOL, Almeida PC, Dodt RCM, Teles NSB. Triagem clínica do processo de doação de sangue: análise da recusa dos doadores. Rev Enferm UFPE On Line [Internet]. 2015 [acesso em 2018 out 15];9(Supl 1):424-30. Disponível em: http://www.repositorio.ufc.br/bitstream/riufc/10795/1/2015_art_nsbteles.pdf doi: 10.5205/reuol.522143270-1-RV.0901supl201522

12. Pereira JR, Sousa CV, Matos EB, Rezende LBO, Bueno NX, Dias AM. To donate or not donate, that is the question: an analysis of the critical factors of blood donation. Ciênc Saúde Colet [Internet]. 2016 [acesso em 2019 dez 24];21(8):2475-84. Disponível em: https://www.scielosp.org/pdf/csc/2016.v21n8/24752484/en doi: 10.1590/1413-81232015218.24062015

13. Freire ACS, Vasconcelos HCA. Doação de sangue: conhecimento, prática e atitude de acadêmicos de enfermagem de uma instituição do interior do Ceará. REME Rev Min Enferm [Internet]. 2013 [acesso em 2020 fev 04];17(2):296-303. Disponível em: http://www.dx.doi.org/10.5935/1415-2762.20130023

14. Öhrner C, Kvist M, Wiberg KB, Diedrich B. Why do young men lapse from blood donation? Vox Sang [Internet]. 2019 [acesso em 2018 set 09];114(6):566-75. Disponível em: https://onlinelibrary.wiley.com/doi/epdf/10.1111/vox.12796 doi: 10.1111/vox.12796

15. World Health Organization (WHO). World blood donor day. Genebra: World Health Organization; 2015.

16. Silva GEM, Valadares GV. Conhecendo os meandros da doação de sangue: implicações para a atuação do enfermeiro na hemoterapia. Rev Bras Enferm [Internet]. 2015 [acesso em 2018 set 09];68(1):32-9. Disponível em: http://www.scielo.br/scielo.php?pid=S003471672015000100032\&script=sci_abstract\&tlng=pt doi: 10.1590/0034-7167.2015680105p

17. Silva AEFA, Pereira JR, Lopes Filho BB. Doação de sangue: a cobertura do jornalismo local e sua contribuição para a formação da opinião pública. RECIIS [Internet]. 2015 [acesso em 2018 jun 12];9(4):116. Disponível em: https://www.reciis.icict.fiocruz.br/index.php/reciis/article/view/1001 doi: 10.29397/reciis.v9i4.1001

18. Calvo-Gonzales E. Hemoglobinas variantes na área médica e no discurso cotidiano: um olhar sobre raça, nação e genética no Brasil contemporâneo. Saúde Soc [Internet]. 2017 [acesso em 2018 out 
07];26(1):75-87. Disponível em: http://www.scielo.br/scielo.php?pid=S010412902017000100075\&script=sci_abstract\&tlng=pt doi: 10.1590/s0104-12902017157821

19. Teles AF, Silva LC, Silva AC, Souza LO, Santos MG, Seibert CS. Hemoglobinas de origem africana em comunidades quilombolas do estado do Tocantins, Brasil. Rev Pan-Amaz Saúde [Internet]. 2017 [acesso em 2018 ago 15];8(1):39-46. Disponível em: http://scielo.iec.gov.br/scielo.php?script=sci_arttext\&pid=S2176-62232017000100039 doi: 10.5123/s217662232017000100006

20. Ramalho AS, Magna LA, Giraldi T. A complexidade da mistura racial no Brasil: a hemoglobina $S$ como marcador étnico nas suas populações. Rev Bras Hematol Hemoter [Internet]. 2006 [acesso em 2018 out 03];28(1):69-70. Disponível em: http://www.scielo.br/scielo.php?script=sci_arttext\&pid=S151684842006000100017 doi: 10.1590/S1516-84842006000100017

21. Murao M, Ferraz MHC. Traço falciforme - heterozigose para hemoglobina S. Rev Bras Hematol Hemoter [Internet]. 2007 [acesso em 2018 ago 08];29(3):223-5. Disponível em: http://www.scielo.br/scielo.php?script=sci_arttext\&pid=S1516-84842007000300006 doi: 10.1590/S151684842007000300006

22. Carlesso L, Guimarães RFS, Silva SL, Santos CF, Viero V, Vieira SV, et al. Estratégias implementadas em hemocentros para aumento da doação de sangue. Rev Bras Promoç Saúde [Internet]. 2017 [acesso em 2018 set 15];30(2):213-20. Disponível em: https://periodicos.unifor.br/RBPS/article/view/5873

\section{Autor correspondente}

Nara Marilene Girardon-Perlini

E-mail: nara.girardon@gmail.com

Endereço: Av. Roraima, 1000. Prédio 26. Sala 1339. Cidade Universitária. Santa Maria - RS. Brasil.

CEP: $97105-900$

\section{Contribuições de Autoria}

\section{1 - Fernanda Duarte Siqueira}

Planejamento do projeto, obtenção, análise e interpretação dos dados, redação do artigo, revisão crítica.

\section{2 - Nara Marilene Oliveira Girardon-Perlini}

Concepção e planejamento do projeto, obtenção, análise e interpretação dos dados, redação do artigo e revisão crítica.

\section{3 - Anelise Levay Murari}

Análise e interpretação dos dados, redação do artigo. 


\section{4 - Carla Tatiana dos Santos Coelho}

Obtenção, análise e interpretação dos dados.

\section{5 - Lauvir Freitas Carneiro}

Obtenção, análise e interpretação dos dados, redação do artigo.

\section{Como citar este artigo}

Siqueira FD, Girardon-Perlini NMO, Murari AL, Coelho CTS, Carneiro LF. Características sociodemográficas e clínicas de adolescentes candidatos à doação de sangue em um hemocentro regional. Rev. Enferm. UFSM. 2020 [Acesso em: Anos Mês Dia]; vol.10 e26: 1-18. DOI:https://doi.org/10.5902/2179769237814 